\title{
A COUNTEREXAMPLE TO THE "FINE" PROBLEM IN PLURIPOTENTIAL THEORY
}

\author{
URBAN CEGRELL AND EVGENY A. POLETSKY
}

(Communicated by Eric Bedford)

\begin{abstract}
In this paper we prove that pointwise values of the non-regularized pluriharmonic measure are not capacities. This answers the question raised by E. Bedford and U. Cegrell.
\end{abstract}

The purpose of this note is to give a counterexample to a problem posed in $[1$, p. 84$]$ and $[2$, p. 52$]$.

Let $D$ be a domain in $\mathbb{C}^{n}, A \subset D$, and $z \in D$. Then the function $\omega(z, A a, D)=\sup \{u(z): u<0$ is plurisubharmonic on $D$ and $u \leq-1$ on $A\}$

is called the pluriharmonic measure of $A$ in $D$.

The "fine" problem is to decide if the set function taking $A$ to $-\omega(z, A, D)$ is a Choquet capacity on subsets of $D$. The axioms to be checked are:

(1) If $K_{j}$ is a decreasing sequence of compact sets in $D$, then

$$
\lim _{j \rightarrow \infty} \omega\left(z, K_{j}, D\right)=\omega\left(z, \bigcap_{j=1}^{\infty} K_{j}, D\right) .
$$

(2) If $A_{j}$ is an increasing sequence of subsets of $D$, then

$$
\lim _{j \rightarrow \infty} \omega\left(z, A_{j}, D\right)=\omega\left(z, \bigcup_{j=1}^{\infty} A_{j}, D\right) .
$$

It is not hard to see that the first axiom is true, since we are looking at the unregularized pluriharmonic measure. Also it was proved in [3] that (2) is true if the sets $A_{j}, j \in \mathbb{N}$, and $A=\bigcup_{j=1}^{\infty} A_{j}$ are compact.

Using some ideas from [4], adapted to this concrete situation, we are going to construct an $F_{\sigma}$-set $A$ for which (2) is false, thus giving a counterexample to the "fine" problem.

Received by the editors May 2, 1994.

1991 Mathematics Subject C'assification. Primary 32F05; Secondary 31C10, $31 \mathrm{C} 15$.

Key words and phrases. Plurisubharmonic functions, capacities. 
Let

$$
\begin{gathered}
D=\left\{z=\left(z_{1}, z_{2}\right) \in \mathbb{C}^{2}:\|z\|^{2}=\left|z_{1}\right|^{2}+\left|z_{2}\right|^{2}<4\right\}, \\
\gamma_{0}=\left\{\left(e^{i \theta}, 0\right) \in \mathbb{C}^{2}: \frac{\pi}{2} \leq \theta \leq \frac{3 \pi}{2}\right\}, \\
\gamma_{j}=\left\{\left(e^{i \theta}, 2^{-j} e^{i \theta}\right) \in \mathbb{C}^{2}:-\frac{\pi}{2} \leq \theta \leq \frac{\pi}{2}\right\}, \\
A_{k}=\bigcup_{j=0}^{k} \gamma_{j}, \quad A=\bigcup_{j=0}^{\infty} \gamma_{j} .
\end{gathered}
$$

Theorem. $\lim _{k \rightarrow \infty} \omega\left(0, A_{k}, D\right)>\omega(0, A, D)=-1$.

Proof. Let $u<0$ be a plurisubharmonic function on $D$ such that $u \leq-1$ on $A$. We fix $\varepsilon>0$ and let $V=\{z \in D: u(z)<-1+\varepsilon\}$. If $j$ is sufficiently large the circles $S_{j}=\left\{\left(\zeta, 2^{-j} \zeta\right):|\zeta|=1\right\}$ are in $V$. Thus $u(0) \leq-1+\varepsilon$ by the maximum principle. Therefore $\omega(0, A, D)=-1$.

Denote by $\Gamma_{0}=\left\{\left(z_{1}, 0\right) \in D\right\}$ and $\Gamma_{j}=\left\{\left(z_{1}, 2^{-j} z_{1}\right) \in D\right\}$. Then $\Gamma_{0}, \Gamma_{j}$, $j \in \mathbb{N}$, are complete pluripolar sets in $D$, and $\gamma_{0} \subset \Gamma_{0}, \gamma_{j} \subset \Gamma_{j}, j \in \mathbb{N}$. We claim that there is a constant $a, 0>a>-1$, such that for $\|z\|=1 / 16$ we have $\omega(z, A, D) \geq a$.

Note first that if $z \in D$ and $z \notin \bigcup_{j=0}^{\infty} \Gamma_{j}$, then $\omega(z, A, D)=0$. Consider the function $v(z)=\omega(z, K, R)$ where $R$ is the disk in $\mathbb{C}$ of radius 3 centered at 0 and $K=\left\{e^{i \theta}:-\pi / 2 \leq \theta \leq \pi / 2\right\}$. Since $K$ is polynomially convex,

$$
a=\inf _{|z| \leq 1 / 4} \omega(z, K, R)>-1 \text {. }
$$

Therefore, $v_{j}(z)=v\left(z_{1}\left(1+2^{-j}\right)-z_{2}\right) \geq a$ when $z=\left(z_{1}, z_{2}\right) \in \Gamma_{j}, j \geq 1$, and $\|z\| \leq 1 / 16$. It is easy to check that $v_{j}=-1$ on $\gamma_{j}$. When $j=0$ we consider $v_{0}\left(z_{1}, z_{2}\right)=v\left(-z_{1}\right)$. Clearly $v_{0}$ satisfies the properties above.

Suppose that $z_{0} \in \Gamma_{m}$ and $\left\|z_{0}\right\|=1 / 16$. Then $z_{0} \notin \Gamma^{m}:=\bigcup_{j \neq m} \Gamma_{j}$, so since each $\Gamma_{j}$ is a complete pluripolar set, for every $\varepsilon>0$ we can find a negative plurisubharmonic function $g$ on $D$ such that $g=-\infty$ on $\Gamma^{m}$ but $g\left(z_{0}\right)>-\varepsilon$. Since $g(z)+v_{m}(z) \leq-1$ on $A$, we see that $\omega\left(z_{0}, A, D\right) \geq a-\varepsilon$ and, since $\varepsilon>0$ is arbitrary, $\omega\left(z_{0}, A, D\right) \geq a$.

We now prove that

$$
\omega\left(0, A_{k}, D\right) \geq a
$$

for any $k$. Since $A_{k}$ is compact in $D$,

$$
\begin{array}{r}
\omega\left(z, A_{k}, D\right)=\sup \{u(z): u<0 \text { is plurisubharmonic and continuous in } D \\
\text { and } \left.u \leq-1 \text { on } A_{k}\right\} .
\end{array}
$$

Therefore, for every $\varepsilon>0$ there is a continuous plurisubharmonic function $u$ on $D$ such that $u \leq 0$ on $D, u \leq-1$ on $A_{k}$, and $u(z) \geq a-\varepsilon$ when $\|z\|=$ 1/16. Define $h(z)=u(z)$ when $\|z\| \geq 1 / 16$ and $h(z)=\max (u(z), a-\varepsilon)$ when $\|z\|<1 / 16$. Then $h(z)$ is negative and plurisubharmonic on $D$ and $h \leq-1$ on $A_{k}$. Thus $\omega\left(z, A_{k}, D\right) \geq h(z)$, so in particular $\omega\left(0, A_{k}, D\right) \geq$ $h(0) \geq a-\varepsilon$, which completes the proof of the theorem. 


\section{REFERENCES}

1. E. Bedford, Survey of pluripotential theory, Several Complex Variables (J. E. Fornaess, ed.) (Proceedings of the Mittag-Leffler Institute, 1987-1988), Princeton Univ. Press, Princeton, NJ, 1993, pp. 48-97.

2. U. Cegrell, Capacities in complex analysis, Vieweg, Braunschweig and Wiesbaden, 1988.

3. _ Capacities and extremal plurisubharmonic functions on subsets of $\mathbb{C}^{n}$, Ark. Mat. 18 (1980), 199-206.

4. E. A. Poletsky, Analytic geometry on compacta in $\mathbb{C}^{n}$, Math $Z$. (to appear).

Department of Mathematics, University of UMEA, S-901 87 UMEA, SWEden

E-mail address: urban. cegrellemathdept .umu. $\mathrm{se}$

215 Carnegie Hall, Syracuse University, Syracuse, New York 13244

E-mail address: eapolotsemailbox.syr.edu 